\title{
Expanded Diagnostic and Therapeutic Options for Cardiac Disease in Duchenne Muscular Dystrophy
}

\author{
Stöllberger Claudia* and Josef Finsterer \\ Krankenanstalt Rudolfstiftung, A-1030 Wien, Österreich, Austria
}

With interest we read the article by Fayssoil et al. about cardiac involvement classification and therapy in patients with Duchenne muscular dystrophy (DMD) [1]. We agree with the authors that search for cardiac involvement and cardiac therapy are important in the care of patients with DMD, however we want to add some comments and suggestions.

Regarding symptoms, it is mentioned that "classical" symptoms of heart failure like exertional dyspnea or symptoms of arrhythmias like palpitations may be absent in DMD because of the limited mobility. Thus, instrumental investigations to assess the cardiac function are important. The authors recommend electrocardiogram, echocardiography and cardiac magnetic resonance imaging. However, the armamentarium of the cardiologists to detect cardiac dysfunction is larger. First, we recommend measurement of the serum levels of brain natriuretic peptide (BNP) which is a robust indicator for cardiac dysfunction, useful for diagnostic purposes and for control of therapeutic effects, as recommended by current heart failure guidelines [2]. Secondly, we suggest that in patients with suspected arrhythmia 24-Holter monitoring or, if the arrhythmias occur only rarely, implantable loop recorders (ILRs) should be considered. ILRs are small devices, implanted or injected subcutaneously in the left side of the chest under local anaesthesia. They continuously record and delete a

\footnotetext{
${ }^{*}$ Correspondence to: Univ. Prof. Dr. Claudia Stöllberger, Steingasse 31/18, A-1030 Wien, Österreich, Austria. Tel.: +43 676403 11 87; Fax: +43 171165 2209; E-mail: claudia.stoellberger@ chello.at.
}

bipolar electrocardiographic signal with no need for intravascular leads and have a battery capable of storing significant events for up to 36 months. Thanks to their ability to register the patients' electrocardiogram during spontaneous symptoms, ILRs have gained popularity and are now recommended as an early diagnostic tool in the evaluation of unexplained syncope [3]. They are also recommended in patients with other neuromuscular disorders [4].

Regarding the pathophysiology of heart failure in DMD, not only the left but also the right ventricle may be involved by systolic dysfunction and fibrotic degeneration of the myocardium. However, in a larger series of DMD patients right ventricular function and ventricular size was relatively preserved [5]. Not only the myocardiocytes but also the cells of the cardiac conduction system may be involved thus leading to arrhythmias and conduction disturbances.

Regarding therapy, ACE inhibitors, betablockers and aldosterone antagonists are recommended, however, low blood pressure may preclude administration of these drugs. Since DMD patients suffer frequently from sinustachycardia which may induce systolic dysfunction, a rate control in these patients should be achieved, although no therapeutic "goal rate", so far, has been established. If betablockers are not tolerated because of arterial hypotension, other substances should be used. Digitalis with its combination of positive inotropic activity with negative chronotropic effects should be discussed as a therapeutic option [6]. A further substance without blood-pressure lowering effects is ivabradine, an I(f) blocker which 
reduces heart rate and improves systolic function. Ivabradine, so far, has been reported to improve heart failure in two patients with DMD who did not tolerate betablockers [7, 8]. Patients with DMD are frequently treated with steroids for muscle weakness, which also seems to improve left ventricular function [9]. In patients with intractable heart failure, implantation of a ventricular assist device (VAD) or even heart transplantation should be considered [10]. There is also a need to mention that cardiac function may improve if severe scoliosis, a frequent complication of affection of the axial muscles in DMD, is stabilised by Unit Rod stabilization.

Overall, management of cardiac disease in DMD not only requires the cardiologist but also the pulmonologist, the orthopedist, physiotherapist, and the neurologist. Diagnosing and treating cardiac disease in DMD requires a comprehensive, multiprofessional approach and the application of all available diagnostic and therapeutic possibilities. Due to the heterogeneity of clinical presentation and variability with progression of the disease, therapy needs to be individualized to optimally meet the individual patient's needs.

\section{CONFLICT OF INTEREST}

The authors have no conflict of interest to report.

\section{REFERENCES}

[1] Fayssoil A, Abasse S, Silverston K. Cardiac Involvement Classification and Therapeutic Management in Patients with Duchenne Muscular Dystrophy. J Neuromuscul Dis. 2017;4(1):17-23. doi: 10.3233/JND-160194
[2] Ponikowski P, Voors AA, Anker SD, Bueno H, Cleland JG, Coats AJ, et al. 2016 ESC Guidelines for the diagnosis and treatment of acute and chronic heart failure: The Task Force for the diagnosis and treatment of acute and chronic heart failure of the European Society of Cardiology (ESC). Eur J Heart Fail. 2016;18(8):891-975. doi: 10.1002/ejhf.592

[3] Galli A, Ambrosini F, Lombardi F. Holter Monitoring and Loop Recorders: From Research to Clinical Practice. Arrhythm Electrophysiol Rev. 2016;5(2):136-43. doi: 10.15 420/AER.2016.17.2

[4] Finsterer J, Stöllberger C, Keller H. Arrhythmia-related workup in hereditary myopathies. J Electrocardiol. 2012; 45(4):376-84. doi: 10.1016/j.jelectrocard.2012.02.003

[5] Mehmood M, Hor KN, Al-Khalidi HR, Benson DW, Jefferies JL, Taylor MD, et al. Comparison of right and left ventricular function and size in Duchenne muscular dystrophy. Eur J Radiol. 2015;84(10):1938-42. doi: 10.1016/j.ejrad. 2015.07.007

[6] Ziff OJ, Kotecha D. Digoxin: The good and the bad. Trends Cardiovasc Med. 2016;26(7):585-95. doi: 10.1016/ j.tcm.2016.03.011

[7] De Benedittis G, Rosa GD, D'Ettorre E, Piscitelli P, Distante A, de Gregorio C. Effect of Ivabradine in dilated cardiomyopathy from Duchenne muscular dystrophy: A chance for slowing progression of heart failure? Int J Cardiol. 2016;223:286-8. doi: 10.1016/j.ijcard.2016.08.135

[8] Finsterer J, Stöllberger C, Berger E. Beneficial effect of ivabradine in dilated cardiomyopathy from Becker muscular dystrophy. Herz. 2012;37(6):702-5.

[9] Mavrogeni S, Papavasiliou A, Douskou M, Kolovou G, Papadopoulou E, Cokkinos DV. Effect of deflazacort on cardiac and sternocleidomastoid muscles in Duchenne muscular dystrophy: A magnetic resonance imaging study. Eur J Paediatr Neurol. 2009;13(1):34-40. doi: 10.1016/j.ejpn. 2008.02.006

[10] Finsterer J, Cripe L. Treatment of dystrophin cardiomyopathies. Nat Rev Cardiol. 2014;11(3):168-79. doi: 10.1038/nrcardio.2013.213 